\title{
A Linear Interpolation Algorithm for Spectral Filter Array Demosaicking
}

\author{
Congcong Wang, Xingbo Wang, and Jon Yngve Hardeberg \\ The Norwegian Colour and Visual Computing Laboratory \\ Gjøvik University College, Gjøvik, Norway \\ \{congcong. wang, xingbo. wang, jon.hardeberg\}@hig.no \\ http://www.colourlab.no
}

\begin{abstract}
Linear interpolation methods have the characteristics of low computational complexity which makes them widely developed in CFA (color filter array) demosaicking. However, the trichromatic nature of colour images enables CFA demosaicking algorithms to take advantage of the luminance-chrominance representation to interpolate the colour planes efficiently and effectively. It seems, however, this does not apply to multispectral images in a straightforward manner. In this paper, we first propose a linear interpolation method for SFA (spectral filter array) demosaicking drawing on the mathematical analysis of mosaicking, demosaicking processes and the idea of residual interpolation. We then compare the performance of the proposed method with that of five other techniques by means of the SSIM index. The result shows that our new algorithm has a good performance with less computing time.
\end{abstract}

Keywords: Multispectral, Demosaicking, Linear, Residual.

\section{Introduction}

Multispectral/hyperspectral imaging is now a promising technology for the capture, analysis and representation of objects' accurate information which is widely used in remote sensing, biological and so on. Inspired by the revolution in color imaging system caused by color filter array (CFA) [3], some research work appears and makes progress in the design of SFA (spectral filter array, a mosaic array comprised of more than three types of filters) and the associated demosaicking algorithms, aimed at a low-cost, portable and efficient multispectral imaging system, as shown in Fig. 1. However, introducing the mosaic technique into multispectral system will lose plenty of spectral information which needs to be estimated in a proper way.

Image demosaicking refers to the interpolation of raw data (CFA samples) 8 to obtain full resolution color images, which is a similar problem in SFA demosaicking. In the last decades, a large amont of CFA based methods have been proposed. According to whether the inter-channel correlation is utilized or not, they can be divided into two main categories [15]. The first one treats each channel separately without making use of inter-channel correlation. The techniques

A. Elmoataz et al. (Eds.): ICISP 2014, LNCS 8509, pp. 151-160, 2014.

(C) Springer International Publishing Switzerland 2014 

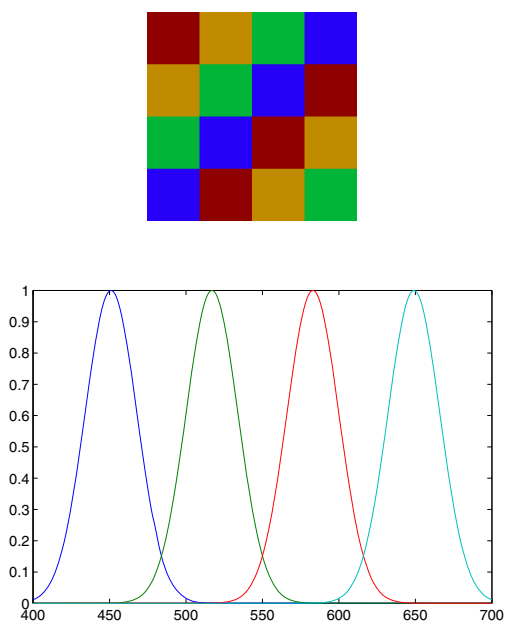
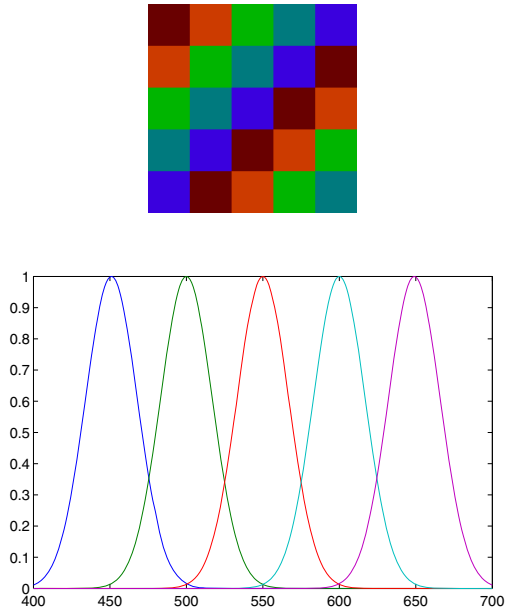

Fig. 1. Spectral filter arrays (left: 4-band, right: 5-band) with corresponding spectral transmittances of the filter sets

include nearest-neighbor interpolation, bilinear/bicubic interpolation [18], spline interpolation [5] and so on. This type of interpolation methods are simple but suboptimal. Exploiting the inter-channel information is of great significance for improving the performance of the demosaicking algorithms. The design of Bayer CFA has the properties that $\mathrm{G}$ channel contains the most information, and can be regard as the luminance channel which has less aliasing. Therefore, most of the methods interpolate the $\mathrm{G}$ channel first, then utilize the interpolated $\mathrm{G}$ channel for the recovery of the chrominance channels (R, B). According to the spatialdomain and frequency domain properties, there are many methods proposed, the detail information can be found in 8]. There are also some other approaches, such as the discrete wavelet transform (DWT) based interpolation, in which the images are transformed into various frequency bands, the high similarity between the high-frequency bands can help to recover the mosaicked images [4.

To our best knowledge, the earliest work on SFA demosaicking is proposed in 2006 by Miao et al. 9], in which they extend the idea of CFA to multispectral imaging by developing generic mosaicking and demosaicking algorithms based on the binary tree theory. Later some more work has been published which focuses more on the demosaicking methods. Baone and Qi [2] explore ways of extending the existing methods to multispectral imaging directly. They treat the demosaicking process as an image restoration task and solve the optimization problem using the gradient descent method which can also reduce the external noise and degradations. Recently, the kernel upsampling and guided filter method is also introduced into multispectral demosaicking in [11] [12]. In [12, they assume 
that the guide image can be generated from the most densely sampled spectral component of SFA, then the other spectral components can be interpolated by the guided filter technique. Another example of extending CFA demosaicking method to SFA demosaicking is the idea Wang et al. proposed in [15]. Most of the demosaicking ideas used in SFA come from the mature CFA (especially Bayer CFA) based methods. In addition, a frequency analysis based multispectral demosaicking method is still under development [14.

Therefore, in this work, we focus on extending some linear methods to multispectral domain. This paper is organized as follows. In section 2 , the proposed method is presented in detail. Result and analysis of the new method as well as other algorithms are shown in section 3. Section 4 concludes the work and suggests directions for future research.

\section{Proposed Method}

In order to design a generic algorithm that can handle the diversified SFA patterns, we need go back to the mathematical analysis of mosaicking and demosaicking as shown in [7. Assume $\mathrm{Y}$ is a full resolution multispectral image and $\mathrm{X}$ is the corresponding SFA image, the mosaicking process can be presented in equation 1:

$$
X=P Y \text {. }
$$

where $\mathrm{P}$ represents the mosaic which converts the original full resolution image $\mathrm{Y}$ to SFA image X. If the superpixels are stacked in a row-wise way, the size of matrix $\mathrm{P}$ is fixed [7. If we can estimate $\mathrm{P}$, the demosaicking process can be written as $\tilde{Y}=P^{-1} X=D X$. It is not sufficient to estimate $\tilde{Y}$ from $\mathrm{P}$ and $\mathrm{X}$. Therefore, a larger kernel contains the information of the neighborhood pixels is required, we use a $n \times n$ window here.

Assume the SFA has 4 channels and we use R channel in Fig. 2 for example. Every $\mathrm{R}$ value is estimated by its neighbors in the $n \times n$ window. Regardless of the size of the image, the size of matrix $\mathrm{D}$ is $n^{2} \times 4 \times 4 . n^{2}$ represents the window's size, $w_{i j}$ are the weights of different neighbors within the window. The first ' 4 ' means there are four types of $R$ values: $R$ values in position $1,2,3$ and 4 which is determined by the SFA pattern. The second ' 4 ' means we have 4 channels in the SFA. The red rectangles of Fig. 2 show the computational process in recovering R1.

If we divide an original full resolution image $\mathrm{Y}$ by the mosaicked image $\mathrm{X}$, we can estimate the parameters of D. D depends on the instinct characters of the SFA pattern, and represents the correlations between one pixel and its neighbouring pixels in the vicinity of $n^{2}$ window. Then the trained $\mathrm{D}$ can be used to estimate $\tilde{Y}$ for other mosaicked images X. Apparently, D is a global matrix which is sensitive to noise and will introduce large residual for a local particular pixel.

In order to reduce the artifacts, we also propose the second step of this method. In paper [6], they propose a residual interpolation idea to replace the color 
$R$ channel of reshaped mosaicked image $X$. e.g. Red rectangle contains the $n^{2}$ neighbour pixels of R1

\begin{tabular}{|l|l|l|l|l|l|l|}
\hline$R 11$ & $R 12$ & $R 13$ & $R 14$ & $R 15$ & $\ldots \ldots$ & $R 1 n^{2}$ \\
\hline$R 21$ & $R 22$ & $R 23$ & $R 24$ & $R 25$ & $\ldots \ldots$ & $R 2 n^{2}$ \\
\hline$R 31$ & $R 32$ & $R 33$ & $R 34$ & $R 35$ & $\ldots \ldots$ & $R 3 n^{2}$ \\
\hline$R 41$ & $R 42$ & $R 43$ & $R 44$ & $R 45$ & $\ldots \ldots$. & $R 4 n^{2}$ \\
\hline
\end{tabular}

\begin{tabular}{|l|l|l|l|l|l|l|}
\hline$R 11$ & $R 12$ & $R 13$ & $R 14$ & $R 15$ & $\cdots \ldots$ & $R 1 n^{2}$ \\
\hline$R 21$ & $R 22$ & $R 23$ & $R 24$ & $R 25$ & $\ldots \ldots$ & $R 2 n^{2}$ \\
\hline$R 31$ & $R 32$ & $R 33$ & $R 34$ & $R 35$ & $\ldots \ldots$ & $R 3 n^{2}$ \\
\hline$R 41$ & $R 42$ & $R 43$ & $R 44$ & $R 45$ & $\ldots \ldots$ & $R 4 n^{2}$ \\
\hline
\end{tabular}
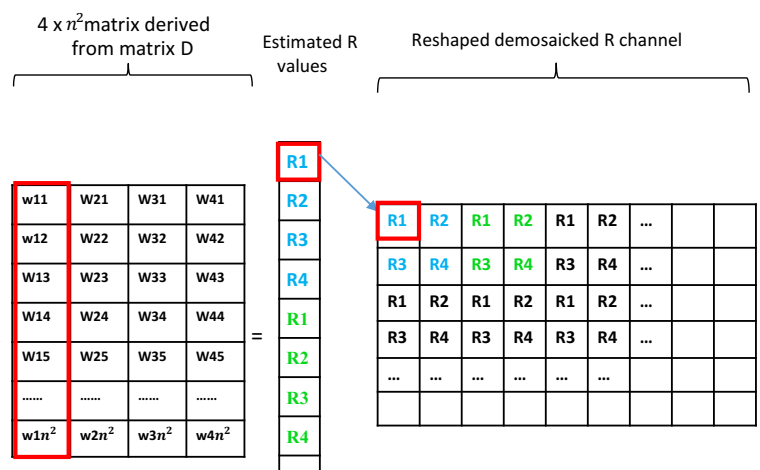

Fig. 2. Illustration of how a full resolution image $\tilde{Y}$ is constructed from a matrix multiplication between mosaicked image $\mathrm{X}$ and the matrix D (use $\mathrm{R}$ channel for example)

difference interpolation in CFA based demosaicking. The residual idea is suitable for multispectral images without other constraints.

In Fig. 3, channel 1 is shown as an example. Firstly, the instinct matrix D is applied to the original mosaicked image, after which the estimated full resolution image $\tilde{Y}$ is obtained by $\tilde{Y}=D X$, called tentative estimate image. Secondly, each tentative estimated channel is subtracted by the original mosaicked channel in order to get the residuals $\Delta$. Then simple linear interpolation method is performed to get the estimated residuals $\Delta^{\prime}$. Finally, the demosaicked image is acquired by adding the estimated residuals to the tentative estimate image.

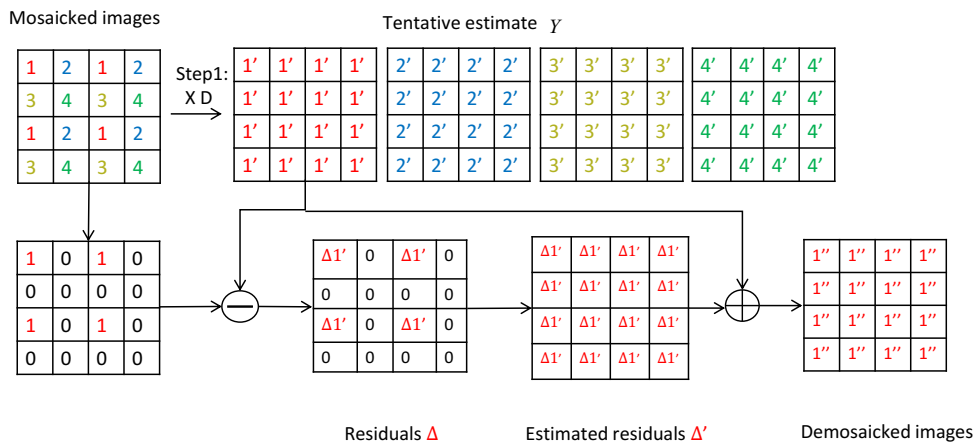

Fig. 3. Framework of the second step of the proposed method 
Table 1. Performance of the algorithms

(a) Results of 4-band SFA demosaicking

\begin{tabular}{|c|c|c|c|c|c|c|}
\hline Images & $\begin{array}{l}\text { Proposed } \\
\text { method }\end{array}$ & DWT 15] & $\begin{array}{l}\text { Binary } \\
\text { tree [10] }\end{array}$ & Bilinear 18 & $\begin{array}{l}\text { Channel } \\
\text { differ- } \\
\text { ence 13 }\end{array}$ & LMMSE 7 \\
\hline fake \& real peppers & 0.9968 & 0.9794 & 0.9981 & 0.4803 & 0.9977 & 0.9768 \\
\hline fake \& real strawberries & 0.9955 & 0.9831 & 0.9927 & 0.3943 & 0.9928 & 0.967 \\
\hline fake \& real sushi & 0.9985 & 0.9926 & 0.9978 & 0.5006 & 0.9972 & 0.9788 \\
\hline fake \& real tomatoes & 0.9974 & 0.9905 & 0.9936 & 0.4826 & 0.9941 & 0.9346 \\
\hline feathers & 0.9902 & 0.9576 & 0.9934 & 0.638 & 0.9907 & 0.9446 \\
\hline flowers & 0.9946 & 0.9663 & 0.9958 & 0.4394 & 0.9929 & 0.8591 \\
\hline glass tiles & 0.988 & 0.9343 & 0.9826 & 0.6728 & 0.9825 & 0.9014 \\
\hline hairs & 0.9983 & 0.9949 & 0.995 & 0.5356 & 0.9956 & 0.9788 \\
\hline jelly beans & 0.9911 & 0.9539 & 0.9864 & 0.463 & 0.9835 & 0.841 \\
\hline oil painting & 0.9833 & 0.9415 & 0.9393 & 0.5377 & 0.9625 & 0.9718 \\
\hline paints & 0.9971 & 0.9716 & 0.9954 & 0.4875 & 0.9943 & 0.9906 \\
\hline photo \& face & 0.9983 & 0.9924 & 0.9972 & 0.5171 & 0.997 & 0.9648 \\
\hline pompoms & 0.9905 & 0.9228 & 0.9928 & 0.5354 & 0.9898 & 0.9934 \\
\hline real \& fake apples & 0.9981 & 0.9939 & 0.9985 & 0.5356 & 0.9981 & 0.9714 \\
\hline real \& fake peppers & 0.9975 & 0.9844 & 0.9973 & 0.5704 & 0.9968 & 0.989 \\
\hline sponges & 0.9931 & 0.9647 & 0.9965 & 0.6363 & 0.9951 & 0.9838 \\
\hline stuffed toys & 0.9972 & 0.9657 & 0.9983 & 0.4678 & 0.9972 & 0.9557 \\
\hline superballs & 0.9935 & 0.9763 & 0.9968 & 0.5903 & 0.9952 & 0.9283 \\
\hline thread spools & 0.9969 & 0.9818 & 0.9888 & 0.4681 & 0.9942 & 0.963 \\
\hline watercolors & 0.9934 & 0.9739 & 0.9848 & 0.5496 & 0.9831 & 0.9891 \\
\hline Average SSIM & 0.994465 & 0.97108 & 0.991055 & 0.52512 & 0.991515 & 0.95415 \\
\hline $\begin{array}{ll}\text { Average Running time } \\
\text { (s) }\end{array}$ & 0.437613 & 4.129056 & 2.013621 & 0.228624 & 3.206924 & 4.545499 \\
\hline
\end{tabular}

(b) Results of 5-band SFA demosaicking

\begin{tabular}{|c|c|c|c|c|c|c|}
\hline Images & $\begin{array}{l}\text { Proposed } \\
\text { method }\end{array}$ & \begin{tabular}{|l|l|} 
DWT & 15 \\
\end{tabular} & $\begin{array}{l}\text { Binary } \\
\text { tree } 10\end{array}$ & Bilinear 18 & $\begin{array}{l}\text { Channel } \\
\text { differ- } \\
\text { ence } 13\end{array}$ & LMMSE 7 \\
\hline fake \& real peppers & 0.9968 & 0.9825 & 0.9973 & 0.4254 & 0.9974 & 0.8946 \\
\hline fake \& real strawberries & 0.987 & 0.9843 & 0.9882 & 0.4162 & 0.9901 & 0.8856 \\
\hline fake \& real sushi & 0.9988 & 0.993 & 0.9968 & 0.5516 & 0.9968 & 0.9614 \\
\hline fake \& real tomatoes & 0.9546 & 0.9911 & 0.9913 & 0.4096 & 0.9925 & 0.9195 \\
\hline feathers & 0.9646 & 0.9645 & 0.9893 & 0.5936 & 0.9916 & 0.8523 \\
\hline flowers & 0.9211 & 0.9729 & 0.9907 & 0.505 & 0.9932 & 0.7683 \\
\hline glass tiles & 0.9674 & 0.9374 & 0.9777 & 0.4959 & 0.9798 & 0.8136 \\
\hline hairs & 0.9988 & 0.995 & 0.9931 & 0.4217 & 0.9953 & 0.971 \\
\hline jelly beans & 0.9543 & 0.9614 & 0.9769 & 0.4301 & 0.9865 & 0.7369 \\
\hline oil painting & 0.9918 & 0.9413 & 0.9344 & 0.4339 & 0.9383 & 0.9444 \\
\hline paints & 0.9906 & 0.974 & 0.9927 & 0.5779 & 0.9902 & 0.9831 \\
\hline photo & 0.9848 & 0.9929 & 0.996 & 0.2991 & 0.9963 & 0.9376 \\
\hline pompoms & 0.9934 & 0.9367 & 0.9892 & 0.5278 & 0.988 & 0.9763 \\
\hline real \& fake apples & 0.9914 & 0.9943 & 0.9979 & 0.4601 & 0.998 & 0.9298 \\
\hline sponges & 0.989 & 0.9866 & 0.9961 & 0.1857 & 0.9965 & 0.964 \\
\hline stuffed toys & 0.9838 & 0.9691 & 0.9957 & 0.4483 & 0.9929 & 0.9706 \\
\hline superballs & 0.9757 & 0.9678 & 0.9969 & 0.4668 & 0.9953 & 0.9245 \\
\hline thread spools & 0.9483 & 0.9771 & 0.9947 & 0.5051 & 0.9912 & 0.8808 \\
\hline thread spools & 0.983 & 0.9817 & 0.9867 & 0.4029 & 0.99 & 0.9246 \\
\hline watercolors & 0.9891 & 0.9745 & 0.9757 & 0.4458 & 0.98 & 0.9757 \\
\hline Average SSIM & 0.978215 & 0.973905 & 0.987865 & 0.450125 & 0.989 & 0.9107 \\
\hline $\begin{array}{l}\text { Average Running time } \\
\text { (s) }\end{array}$ & 0.513189 & 5.292156 & 2.485644 & 0.246373 & 5.687782 & 5.527632 \\
\hline
\end{tabular}




\section{Experimental Result}

Due to the impracticability of realizing customized mosaics, we conducted experiments on a simulated platform [15. The platform uses a hyperspectral image as an approximation to an irradiance images which are spatially sampled and contain rich spectral information. A simulated SFA then spectrally filters the irradiance image, and a simulated sensor with specified spectral sensitivities follows and produces the mosaic multispectral image. Here comes the demosaicking algorithm that recovers the lost information and reproduces a full resolution multispectral image, namely a demosaicked image. Through certain linear regression methods, it is viable to estimate the spectral reflectance or irradiance of each pixel, thus obtaining a hyperspectral estimate of the demosaicked image. The performance of demosaicking algorithms can be evaluated at either multispectral or hyperspectral stage.

The CAVE hyperspectral image database [17. was used in the experiment, which contains 32 reflectance images of real-world materials and objects. This images comprise 31 bands ranging from $400 \mathrm{~nm}$ to $700 \mathrm{~nm}$ with an interval of 10 $\mathrm{nm}$, which defines the spectral range and accuracy of the experiments. An image named 'balloons' was employed for training purpose, and another 20 images selected from CAVE database are used for testing purpose. The images are then lit by the CIE D65 illuminant.

In this work, we experimented with two given types of SFAs, i.e., a 4-band SFA and a 5-band SFA, as shown in Fig. 1. The spectral transmittances of the filters are set so that the peak transmittances sample the visible spectrum evenly. In order to simplify the simulation, an ideal sensor with constant spectral sensitivities across the spectrum was utilised.

For the sake of comparison, we also implemented, in addition to the proposed method, another 5 techniques of multispectral demosaicking including bilinear interpolation [18, channel difference interpolation extended from the color difference method [13], LMMSE method [7] based on constant difference, binary tree based demosaicking [10, and discrete wavelet transformed based approach [15.

Please note that both of the SFAs were designed artificially. As the binary tree based approach works only with mosaic patterns that the accompanying method generates [9], the patterns for this method is slightly different from the SFAs shown in Fig. 11. And in the channel difference interpolation, the third filter is considered as the reference channel as the peak transmittances lie at about $550 \mathrm{~nm}$ for both filter sets used in the two SFAs.

There exists a variety of image quality evaluation methods to assess the performance of interpolation algorithm: PSNR (peak signal-to-noise ratio), SSIM (structural similarity) index, color appearance models and so on. Here, we use the SSIM metric. The SSIM index defines structural information in an image as attributes that represent the structure of the objects in the scene, independent of the average luminance and contrast. The index is based on a combination of luminance, contrast, and structure comparison. A value of 1 means perfect match between the two input images. Detailed information and analyses of SSIM is shown in [16]. To that end, the original and restored hyperspectral have to be 
converted to the sRGB colour space first with corresponding colour matching functions.

The SSIM values of the 20 images for 4-band SFA demosaicking is shown in Table 1(a), the corresponding plot is shown in Fig. 4 . Table 1(b) and Fig. 5] present the result of 5-band SFA demosaicking.

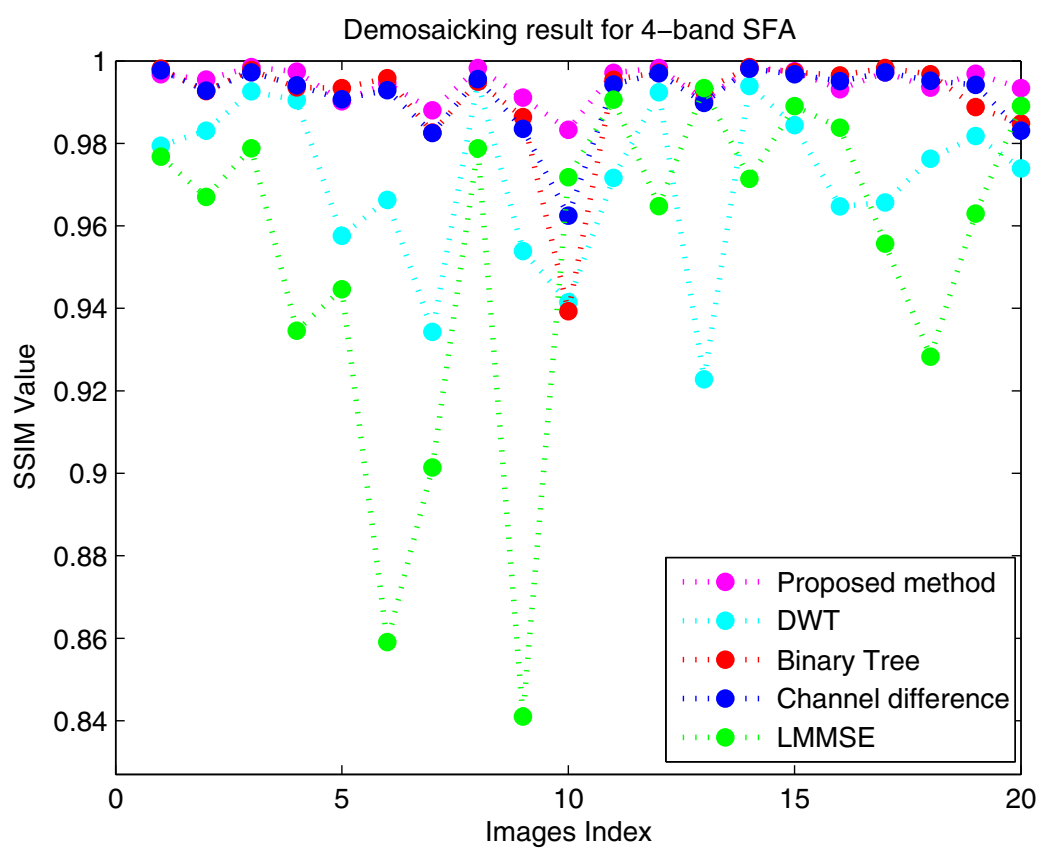

Fig. 4. SSIM index values of the 4-band SFA

The overall result of 5-band SFA demosaicking is not as good as 4-band's case mainly because of the coarser spatial distribution.

In general, the binary tree based method performs fairly well, and can be seen as a benchmark. However, it cannot be compared directly with other methods as the mosaics are not the same.

Our proposed method outperformed the binary tree approach and achieved the best performance for 4-band SFA, because it is not sensitive to the contents of different images. Although, the less information contained in a local window can exaggerate the role of noise which results in the decline of SSIM values for 5-band SFA, it outperforms most of the other methods except binary tree method.

In spite of its simplicity, the channel difference based interpolation [13] yielded promising results especially in the case of the 5-band SFA demosaicking. This suggests that the images tested possess a great deal of smooth chromatic transition. 


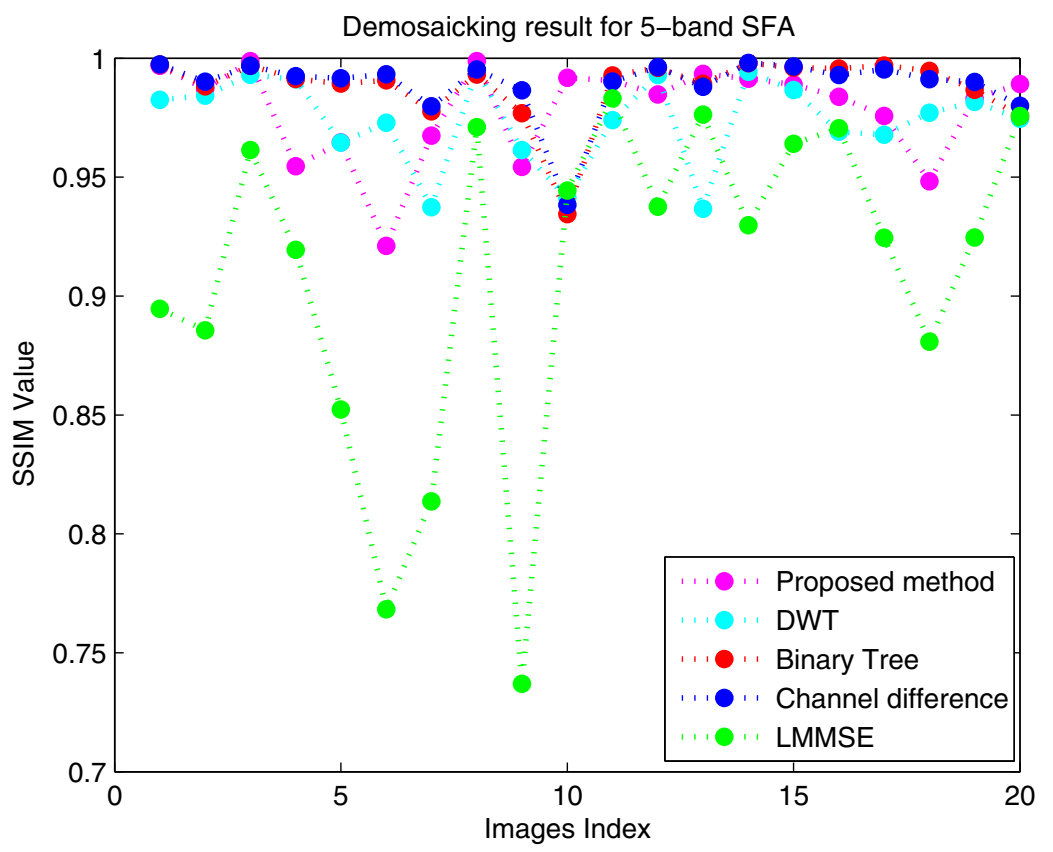

Fig. 5. SSIM index values for the 5-band SFA

The inferior results of DWT based approach [15] may indicate that the interchannel correlation in high-frequency region of the images is not sufficiently high. Besides, the different signatures of each band will reduce the smoothness of inter-band edge information, thus resulting in a worse performance for the 5-band SFA.

The LMMSE method [7] needs more strict assumption, for example the secondorder directional Laplacian filter of Adams and Hailton [1] requires constant color difference in either horizontal or vertical direction.

As expected, bilinear interpolation is the worst in this comparison, however the extremely low SSIM indices are worth further investigations.

Considering the computational efficiency, our proposed method only costs 0.5 seconds on average which shows great improvement compared to all the other methods. Next comes the binary tree method whose time consumption is 2.5 seconds on average, the other methods cost around 5 seconds. Also increasing number of bands led to higher computational cost.

\section{Conclusion}

The assumptions on which a variety of CFA demosaicking methods are based are very often not applicable to the multispectral domain [10]. As an example, the luminance-chrominance color space used for CFA pattern is senseless for 
SFA demosaicking, as a result of the difficulties in extracting luminance and chrominance information separately in multispectral images.

In this paper, we propose a linear method that combine linear Wiener estimation and an interpolation on residual channels in the context of multispectral demosaicking. The latter may overcome the error introduced by the former's sensitivity to noise. This method yielded an average SSIM value of 0.994465 for a 4-band SFA and 0.978215 for a 5-band SFA with the running time of about 0.5 seconds.

This method has several advantages. First, its dependency on the mosaic design is not as high as the other methods. Second, the residual interpolation is of high scalability. Third, the computation efficiency is improved greatly in comparison with other methods. Certainly this method has some disadvantages as well. It requires a priori information and it is sensitive to noise.

The SFA patterns were randomly designed in this article. The rules of designing an efficient SFA with high inter-channel correlation merit further development. In turn, these rules can be used to design homologous demosaicking algorithms. From the performance of the binary tree based interpolation, we can conclude that the relation between mosaic design and demosaicking is important. Therefore, for multispectral demosaicking, it is beneficial to consider mosaic design and demosaicking algorithms as a whole as suggested by Miao et al. [9] [10].

\section{References}

1. Adams, Jr., J.E., Hamilton, Jr., J.F.: Adaptive color plane interpolation in single sensor color electronic camera. Patent, US 5652621 (July 1997)

2. Baone, G.A., Qi, H.: Demosaicking methods for multispectral cameras using mosaic focal plane array technology. In: Proc. SPIE, vol. 6062 (January 2006)

3. Bayer, B.E.: Color imaging array. Patent, US 3971065 (July 1976)

4. Chen, L., Yap, K.H., He, Y.: Color filter array demosaicking using wavelet-based subband synthesis. In: Proceedings of the 12th IEEE International Conference on Image Processing (ICIP), vol. 2, pp. II-1002-5 (September 2005)

5. Hou, H., Andrews, H.: Cubic splines for image interpolation and digital filtering. IEEE Transactions on Acoustics, Speech and Signal Processing 26(6), 508-517 (1978)

6. Kiku, D., Monno, Y., Tanaka, M., Okutomi, M.: Residual interpolation for color image demosaicking. In: Proceedings of the 20th IEEE International Conference on Image Processing (ICIP), pp. 2304-2308 (September 2013)

7. Chaix de Lavarène, B., Alleysson, D., Hérault, J.: Practical implementation of lmmse demosaicing using luminance and chrominance spaces. Computer Vision and Image Understanding 107(1), 3-13 (2007)

8. Li, X., Gunturk, B., Zhang, L.: Image demosaicing: A systematic survey. In: Pearlman, W.A., Woods, J.W., Lu, L. (eds.) Visual Communications and Image Processing 2008, vol. 6822. SPIE (2008)

9. Miao, L., Qi, H., Ramanath, R.: Generic MSFA mosaicking and demosaicking for multispectral cameras. In: Proc. SPIE, vol. 6069 (January 2006)

10. Miao, L., Qi, H., Ramanath, R., Snyder, W.E.: Binary tree-based generic demosaicking algorithm for multispectral filter arrays. IEEE Transactions on Image Processing 15(11), 3550-3558 (2006) 
11. Monno, Y., Tanaka, M., Okutomi, M.: Multispectral demosaicking using adaptive kernel upsampling. In: Proceedings of the 18th IEEE International Conference on Image Processing (ICIP), pp. 3157-3160. Brussels (September 2011)

12. Monno, Y., Tanakaa, M., Okutomia, M.: Multispectral demosaicking using guided filter. In: Battiato, S., Rodricks, B.G., Sampat, N., Imai, F.H., Xiao, F. (eds.) Digital Photography VIII. Proc. of SPIE, Burlingame, California, USA, vol. 8299, pp. 82990O-1-82990O-7 (January 2012)

13. Ramanath, R., Snyder, W.E., Bilbro, G.L., Sander III, W.A.: Demosaicking methods for bayer color arrays. Journal of Electronic Imaging 11(3), 306-315 (2002)

14. Simon, P.M.: Single Shot High Dynamic Range and Multispectral Imaging Based on Properties of Color Filter Arrays. master thesis, University of Dayton (2011)

15. Wang, X., Thomas, J.B., Hardeberg, J.Y., Gouton, P.: Discrete wavelet transform based multispectral filter array demosaicking. In: Proc. Colour and Visual Computing Symposium (CVCS), pp. 1-6 (September 2013)

16. Wang, Z., Bovik, A., Sheikh, H., Simoncelli, E.: Image quality assessment: from error visibility to structural similarity. IEEE Transactions on Image Processing 13(4), 600-612 (2004)

17. Yasuma, F., Mitsunaga, T., Iso, D., Nayar, S.K.: Generalized assorted pixel camera: Post-capture control of resolution, dynamic range and spectrum. Tech. rep., Department of Computer Science, Columbia University CUCS-061-08 (November 2008), http://www.cs.columbia.edu/CAVE/databases/multispectral/

18. Yu, W.: Adaptive cubic convolution interpolation and sequential filtering for color demosaicing of bayer pattern image sensors. In: Proc. SPIE 5909, Applications of Digital Image Processing XXVIII, p. 590915 (2005) 